\title{
Algorithms for Discriminating Between Biochemical Reaction Network Models: Towards Systematic Experimental Design
}

\author{
Antonis Papachristodoulou and Hana El-Samad
}

\begin{abstract}
We develop a methodology for distinguishing between biochemical reaction network models for biological systems modeled by nonlinear ordinary differential equations. We show how to choose the 'best' initial condition direction (the dependent variables, the state) so as to maximize the discrepancy between the outputs of possible network models. This information could then be used to design new experiments with the hope that some of the networks would be invalidated from experimental data. The methodology is applied to discriminate between models of the network underlying chemoattractantinduced movement of Dictyostelium cells.
\end{abstract}

\section{INTRODUCTION}

Advances in molecular biology that made the experimental probing of relationships between microscopic processes possible have recently spurred a large interest in mathematical methods as tools to study biological networks. It is becoming increasingly appreciated that these tools can be instrumental in distinguishing between many plausible interconnections in these networks, such as direct and indirect links between molecular components. Mathematical modeling can then be efficiently used to guide experimental investigations and further help delineate the specific architectures of the biochemical networks under study.

Models of biochemical networks are constructed either following a top-down or bottom-up approach. In the former methodology, a family of appropriate models is usually built as the result of the processing and analysis of data collected from high throughput experiments. In the latter approach simple models that describe the phenomenological behavior of the system are proposed, and as data and other information is collected, the models are refined and become more complex. In the framework of top-down model construction, many methods have been proposed to reverse engineer the "map" of interconnections that occurs between chemical species in a biochemical network [3], [11], [2], [5], [15], [12]. Naturally, all of these techniques result in a model that can reproduce the available data within experimental errors. This, however, does not necessarily imply that other, structurally different models cannot account for the experimental observations. Often times, the existing knowledge about these systems, gained through prior experimentation, can help narrow down the set of possible alternative models.

This material is based upon work supported by the Sandler Family Foundation.

A. Papachristodoulou is with the Department of Engineering Science, University of Oxford, Parks Road, Oxford, OX1 3PJ, U.K. antonis@eng.ox.ac.uk.

H. El Samad is with the Department of Biochemistry and Biophysics and the California Institute for Quantitative Biomedical Research at the University of California, San Francisco, 1700 4th Street, San Francisco, CA 94158, U.S.A. helsamad@biochem. ucsf . edu.
The question then arises: How can one differentiate between the remaining ones? A powerful use of the available models is then to suggest the next experiment that maximizes the difference between the dynamical behaviors of the proposed models, with the hope that the new data obtained through the rationally designed new experiment will be used to invalidate some of them. Such iterations between modeling and experiments is precisely the kind of synergy that one hopes to achieve in the nascent field of Systems Biology. Previous work in this area can be found in [1], [6], [4].

In this paper we describe a first step in this direction: we propose a methodology to choose algorithmically the initial conditions that will maximize the $L_{2}$ norm of the difference in the output of different plausible nonlinear ODE models of the same biochemical system. Naturally, to set a sound basis for comparison between different models, they must structurally give rise to similar qualitative behavior, even if they have different parameters or different structure. This would be the case if, given network models, parameters were being fit so that the resulting models represent the same data.

The structure of the paper is as follows. In Section II, we recall previous results on linear systems, which we adapt to answer the questions of interest in this paper. In section III, we show how storage function arguments along with the sum of squares decomposition can be used to answer the same questions for nonlinear systems and provide algorithms for choosing the initial conditions that would yield maximum discrepancy between two nonlinear systems. In Section IV, we look at the chemoattractant-induced response of Dictyostelium cells and show how our methodology can be applied to design experiments that distinguish between different models for this system.

\section{BACKGROUND}

\section{A. Notation}

We denote by $\mathbb{R}^{n}$ the $n$-dimensional Euclidean space. For $x=\left(x_{1}, \ldots, x_{n}\right)$ we let $\mathbb{R}[x]$ be the ring of polynomials in $x$ with real coefficients. We say $p(x) \in \mathbb{R}[x]$ is non-negative if $p(x) \geq 0$ for all $x \in \mathbb{R}^{n}$. Moreover, we say $p(x) \in \Sigma_{m}$ is a Sum of Squares of degree $2 m$ if there exist $h_{i}(x) \in \mathbb{R}[x]$, $i=1, \ldots, M$ such that $p(x)=\sum_{i=1}^{M} h_{i}^{2}(x)$.

\section{B. Problem Formulation}

Consider the system of state dimension $2 n$ described by:

$$
\begin{aligned}
\frac{d x}{d t} & =f(x)+g(x) u, \\
y & =h(x)
\end{aligned}
$$


where $x \in \mathbb{R}^{2 n}, u \in U \subseteq \mathbb{R}^{m}, f: \mathbb{R}^{2 n} \rightarrow \mathbb{R}^{2 n}$ is locally Lipschitz and $g: \mathbb{R}^{2 n} \rightarrow \mathbb{R}^{2 n \times m}$ is the input function which we assume is continuous. For $y \in \mathbb{R}^{k}$ the output function $h: \mathbb{R}^{2 n} \rightarrow \mathbb{R}^{k}$ is also assumed to be continuous.

For the purpose of model discrimination that is the subject of this paper, we are concerned with systems of the form (1-2) but of a specific structure. Namely, we assume that the $2 n$-dimensional system is comprised of two subsystems which are being compared, each of dimension $n$. The output function $h$ is then a function of the difference of the corresponding states (we take that to be the difference itself in the sequel) and satisfies $h(0)=0$. In particular, we have:

$$
\begin{aligned}
\frac{d}{d t}\left[\begin{array}{l}
x_{1} \\
x_{2}
\end{array}\right] & =\left[\begin{array}{l}
f_{1}\left(x_{1}\right) \\
f_{2}\left(x_{2}\right)
\end{array}\right]+\left[\begin{array}{l}
g_{1}\left(x_{1}\right) \\
g_{2}\left(x_{2}\right)
\end{array}\right] u, \\
y & =h\left(x_{1}-x_{2}\right)
\end{aligned}
$$

Define $x=\left[\begin{array}{ll}x_{1} & x_{2}\end{array}\right]^{T}$ and $f(x)=\left[\begin{array}{ll}f_{1}\left(x_{1}\right) & f_{2}\left(x_{2}\right)\end{array}\right]^{T}$, where $x_{1}, x_{2} \in \mathbb{R}^{n}$. We will also use $x_{1 k}, k=1, \ldots, n$ to denote the state variables in model 1, etc. For this system, and for $u=0$, we want to know what initial conditions $x_{1}(0)=x_{2}(0)$ would result in the largest 2-norm for $y$.

\section{Linear Systems}

For the simpler case of linear systems, this question can be readily answered using existing tools. Consider a linear system of the form

$$
\begin{aligned}
\frac{d x}{d t} & =A x+B u \\
y & =C x
\end{aligned}
$$

where

$$
A=\left[\begin{array}{cc}
A_{1} & 0 \\
0 & A_{2}
\end{array}\right], \quad B=\left[\begin{array}{c}
B_{1} \\
B_{2}
\end{array}\right], \quad C=\left[\begin{array}{ll}
I & -I
\end{array}\right]
$$

We are interested in the initial condition $x_{1}(0)=x_{2}(0)$ for the unforced system $u=0$ that would result in maximum $\|y\|_{2}$. Recall that without the restriction that $x_{1}(0)=x_{2}(0)$, the direction on which the maximum $\|y\|_{2}$ is obtained can be found by the following procedure:

1) Find the solution $P \succeq 0$ to the following Observability Lyapunov equation:

$$
A^{T} P+P A+C^{T} C=0
$$

The solution $P \succeq 0$ is called the Observability Grammian.

2) Find the eigenvector $\hat{x}$ corresponding to the largest eigenvalue $\bar{\lambda}$ of $P$, i.e., find $\hat{x}$ and $\bar{\lambda}$ such that

$$
P \hat{x}=\bar{\lambda} \hat{x} .
$$

It can then be shown that $x(0)=\hat{x}$ would be the initial condition that would result in the largest output energy. However $x(0)$ chosen in this way does not ensure that $x_{1}(0)=x_{2}(0)$, i.e., the requirement that the two subsystems should have the same initial condition. To enforce this condition, we have the following Proposition.
Proposition 1: Consider system (4-5) with $A_{1}, A_{2}$ Hurwitz so that there exists a solution $P \succeq 0$ to the following Lyapunov Equation:

$$
A^{T} P+P A+C^{T} C=0
$$

Partition $P$ as follows

$$
P=\left[\begin{array}{ll}
P_{11} & P_{12} \\
P_{12}^{T} & P_{22}
\end{array}\right]
$$

where $P_{i j} \in \mathbb{R}^{n \times n}$. Then, $x_{1}(0)=x_{2}(0)$ such that $\left\|x_{i}(0)\right\|=1$ for which $\|y\|_{2}^{2}=\int_{0}^{\infty} \sum\left(x_{1 i}(t)-x_{2 i}(t)\right)^{2} d t$ is maximum is the normalized eigenvector corresponding to the largest eigenvalue of the matrix

$$
Q=P_{11}+P_{22}+P_{12}+P_{12}^{T} .
$$

Proof: For system (4-5) we have:

$$
\begin{aligned}
y & =C e^{A t} x_{0}=\left[\begin{array}{ll}
I & -I
\end{array}\right]\left[\begin{array}{cc}
e^{A_{1} t} & 0 \\
0 & e^{A_{2} t}
\end{array}\right]\left[\begin{array}{l}
x_{0} \\
x_{0}
\end{array}\right] \\
& =\left(e^{A_{1} t}-e^{A_{2} t}\right) x_{0},
\end{aligned}
$$

and therefore

$$
\begin{aligned}
\|y\|_{2}^{2}= & x_{0}^{T} \int_{0}^{\infty}\left(e^{A_{1}^{T} t}-e^{A_{2}^{T} t}\right)\left(e^{A_{1} t}-e^{A_{2} t}\right) d t x_{0} \\
= & x_{0}^{T} \int_{0}^{\infty} e^{A_{1}^{T} t} e^{A_{1} t} d t x_{0}+x_{0}^{T} \int_{0}^{\infty} e^{A_{2}^{T} t} e^{A_{2} t} d t x_{0} \\
& -x_{0}^{T} \int_{0}^{\infty} e^{A_{1}^{T} t} e^{A_{2} t} d t x_{0}-x_{0}^{T} \int_{0}^{\infty} e^{A_{2}^{T} t} e^{A_{1} t} d t x_{0} \\
= & x_{0}^{T}\left(P_{11}+P_{22}+P_{12}+P_{12}^{T}\right) x_{0}
\end{aligned}
$$

where the $P_{i j}$ are the partitions of $P$ as in the statement of the Proposition, and solve the corresponding Lyapunov equations. Therefore the eigenvector corresponding to the largest eigenvalue of $Q$ is indeed the direction for which $\|y\|_{2}^{2}$ is maximized.

In the next section we will discuss how we can transfer these ideas to nonlinear systems and use the Sum of Squares technique to answer the question algorithmically.

\section{MAin Results FOR NONLINEAR Systems}

Our approach to treat nonlinear systems is based on the use of Storage functions and dissipativity theory [17], [18] along with the Sum of Squares decomposition.

Theorem 2: Consider system (1-2), and let $f(0)=0$. Let there exist a continuously differentiable function $S(x)$ : $\mathbb{R}^{n} \rightarrow \mathbb{R}$ satisfying:

$$
\begin{aligned}
& S(x) \geq 0, \quad S(0)=0, \\
& -\frac{\partial S}{\partial x} \dot{x}+w(x, y) \geq 0 .
\end{aligned}
$$

Then the system is dissipative with respect to the supply function $w(x, y)$.

In the above theorem, the differential version of the dissipation inequality holds. Indeed, through integration of 
condition (9) we get:

$$
\begin{aligned}
-\int_{0}^{T} w(x(t), y(t)) d t & \leq-\int_{0}^{T} \frac{\partial S}{\partial x} \dot{x} d t \\
=-\int_{0}^{T} \frac{d S(x)}{d t} d t & =-S(x(T))+S(x(0)) .
\end{aligned}
$$

The above method can be used, for example, to estimate upper bounds on the $L_{2}$ norm of the system output when the initial condition is $x(0)$ and $u=0$.

For system descriptions of the form (3), we assume that each subsystem has an asymptotically stable equilibrium point. We translate the system coordinates so that zero is an equilibrium point and restrict our attention in some region of the zero equilibrium, which we define as:

$$
D=\left\{x \in \mathbb{R}^{2 n} \mid\left\|x_{1}\right\|_{2}^{2} \leq 1,\left\|x_{2}\right\|_{2}^{2} \leq 1\right\}
$$

It is then possible to answer the question of finding the 'best' initial condition direction that would maximize the discrepancy between the subsystem outputs for zero input using Storage function arguments:

Proposition 3: Consider system (3) for which we assume that $f_{1}(0)=f_{2}(0)=0$ and $u=0$. Suppose there exists a function $S \geq 0, S: \mathbb{R}^{2 n} \rightarrow \mathbb{R}$ with $S(0)=0$ such that the following holds in a region $D$ around the origin:

$$
-\frac{\partial S}{\partial x} f(x)-h^{T} h \geq 0, \text { for } x \in D
$$

Then $\|y\|_{2}^{2} \leq S(x(0))$ if the system is released from an initial condition $x(0)$ inside the largest level set of $S$ that fits in $D$.

Proof: Consider an initial condition $x(0)$ that is inside the largest level set of $S$ that can fit in $D$. Integrating condition (11) from 0 to $T$ we get:

$$
\int_{0}^{T} h(x(t))^{T} h(x(t)) d t \leq S(x(0))-S(x(T)) .
$$

We now let $T \rightarrow \infty$ and the above becomes

$$
\|y\|_{2}^{2} \triangleq \int_{0}^{\infty} h(x(t))^{T} h(x(t)) d t \leq S(x(0)),
$$

as $S(x(\infty)) \geq 0$ by construction. Since the derivative condition is valid within the region $D$ and level sets of $S$ are invariant, initial conditions that are inside the largest level set of $S$ that can fit in $D$ satisfy $\|y\|_{2}^{2} \leq S(x(0))$.

Note that $S$ in the Proposition satisfies an inequality condition for its derivative, whereas the corresponding condition in the linear case (and hence the linearization of the system) is obtained by solving a Lyapunov equation and is thus 'optimal'. In the next section we discuss a heuristic for getting close to 'optimal' $S$.

Proposition 3 and Proposition 1 are related, but what is missing in Proposition 3 is the condition that the two subsystems are initialized from the same initial condition. Once such an $S$ is constructed using the above programme, we can proceed to find the input direction $\hat{x}(0)$ inside the largest level set of $S$ that can fit in $D$, and which will maximize the 2 -norm of the output $y$ while ensuring that $\hat{x}_{1}(0)=\hat{x}_{2}(0)$. Recall that in the linear case, such $\hat{x}(0)$ corresponds to the eigen-direction with the largest eigenvalue of the matrix $Q$ in (7), i.e., in the direction of the largest semi-axis of the related ellipsoid. In the nonlinear case, this is the point where a level set of $\tilde{S}$ (defined below) would first 'touch' the region $D$. For this, we can solve the following optimization problem:

$$
\begin{array}{cl}
\max & \gamma \\
\text { s.t. } & \tilde{S}\left(\hat{x}_{1}\right)-\gamma \leq 0, \\
& \left\|\hat{x}_{1}\right\|_{2}^{2}=1 .
\end{array}
$$

where $\tilde{S}\left(\hat{x}_{1}\right)=S\left(\hat{x}_{1}, \hat{x}_{1}\right)$. This procedure resembles the one followed in Proposition 1.

Computationally, constructing $S$ and finding the 'best' initial condition $\hat{x}$ is not an easy task. However we can use the Sum of Squares decomposition and semidefinite programming for this purpose. We describe these techniques in the following section.

\section{A. Computational Details}

Constructing a function $S$ that satisfies the non-negativity conditions in Proposition 3 above is a difficult task. In particular, testing non-negativity of a polynomial function is NP-hard. However, such an $S$ can be constructed algorithmically using the Sum of Squares decomposition and Semidefinite programming, as a sufficient condition for nonnegativity. To use the sum of squares technique, we restrict $S$ and the system description to be polynomial, and relax the non-negativity conditions to the existence of a sum of squares decomposition. First, we introduce some terminology relevant to the sum of squares techniques.

If $p(y) \in \Sigma$, then immediately $p(y) \geq 0$ for all $y$. The converse is not always true, except for univariate polynomials, quadratic forms and tertiary quartic forms. While testing if $p(y) \geq 0$ is NP-hard, testing if $p(y) \in \Sigma$ is equivalent to a semidefinite programme (SDP) [7], and hence is worst-case polynomial-time verifiable. In particular, $p(y)$ being SOS is equivalent to the existence of a vector of monomials $Z(y)$ and a semidefinite matrix $Q \succeq 0$ such that

$$
p(y)=Z(y)^{T} Q Z(y) .
$$

The related SDP can be formulated efficiently and the solution can be retrieved using SOSTOOLS [10], which uses semidefinite solvers such as SeDuMi [13] to solve it.

We are now in a position to address the task of constructing an $S$ that satisfies the conditions in Proposition 3. Indeed, all of these conditions are easily enforced in a Sum of Squares programming framework. In total, the following sum of squares programme can be used to construct $S$ :

Given $f_{1}\left(x_{1}\right), f_{2}\left(x_{2}\right)$, and the set description $D$, Find $S(x), \sigma_{1}\left(x_{1}\right), \sigma_{2}\left(x_{2}\right)$ all $\operatorname{SOS}$

such that

$$
\begin{aligned}
& \quad \begin{array}{l}
S(0)=0 \\
-\frac{\partial S}{\partial x} f(x)
\end{array}-h^{T} h+\sigma_{1}(x)\left(\left\|x_{1}\right\|_{2}^{2}-1\right) \\
& \quad+\sigma_{2}(x)\left(\left\|x_{2}\right\|_{2}^{2}-1\right) \text { is SOS. }
\end{aligned}
$$


It is important to note that there is no unique $S$ that satisfies the above conditions. However a heuristic to find the 'best' $S$ is to minimize the trace of matrix $Q$ in (13) in the sum of squares description for $S$.

Furthermore, in order to find a point $\hat{x}_{1}=\hat{x}_{2}$ that would maximize the $L_{2}$ norm of the output, we can solve the following sum of squares programme, which is related to the nonlinear programme (12):

$$
\begin{aligned}
\max & \gamma \\
\text { s.t. } & \tilde{S}\left(\hat{x}_{1}\right)-\gamma+p\left(\hat{x}_{1}\right)\left(\left\|\hat{x}_{1}\right\|_{2}^{2}-1\right) \text { is SOS } \\
& p\left(\hat{x}_{1}\right) \text { a polynomial. }
\end{aligned}
$$

The point $\hat{x}_{1}$ can be obtained from the dual solution of this semidefinite programme, and is exact when the dual variable has rank 1. Let us apply these methods to a case study.

\section{Case Study: Dictyostelium Discoideum Chemotaxis}

\section{A. The Biology of Dictyostelium Discoideum Chemotaxis}

Chemotaxis refers to the directional migration of cells away or towards a chemical stimuli. This process can be found and is of substantial importance both for singlecelled and multicellular organisms. For example, bacteria find food (e.g. glucose) by swimming towards the highest concentration of food molecules [16]. Neutrophils that are our body's first line of defense against bacterial infections recognize chemicals produced by bacteria in a cut or scratch and migrate "toward the smell" [14]. Here, we look at the chemotaxis properties of the best studied chemotactic amoeboid organism Dictyostelium Discoideum [8]. Dictyostelium is chemotactic for bacteria, but has been extensively studied for its ability to climb gradients of the nucleotide cAMP, a signalling molecule involved in the development of the slug. Dictyostelium normally live as unicellular amoebas but, upon starvation, aggregate to form a multicellular organism. The possibility to form such multicellular structures is precisely mediated by chemotaxis. Upon starvation, Dictyostelium cells acquire the ability to synthesize cAMP. Subsequent secretion of the cAMP molecules into the medium directs the chemotactic cells into the aggregate. The chemoattractantinduced response in Dictyostelium cells starts by the binding of extracellular cAMP to cAMP specific receptors on the cell surface, leading to a series of signal transduction events that cause localized F-actin polymerization at the site of the plasma membrane closest to the chemoattractant. F-actin forms the cytoskeleton - a three-dimensional network inside an eukaryotic cell - and as such provides mechanical support for the cell, determines the cell shape, and enables cell movements through the formation of a pseudopod that allows the motility of the Dictyostelium cell. For Dictyostelium as for a host of other chemotactic organisms, it was experimentally demonstrated that induction of chemotaxis through a step input of chemoattractant causes a transient response in the chemotactic proteins, after which they return to their pre-stimulus values. This property has been termed "perfect adaptation", and as a result of a direct application of the Internal Model Principle, can be shown to be the unique outcome of the presence of integral action in the chemotactic
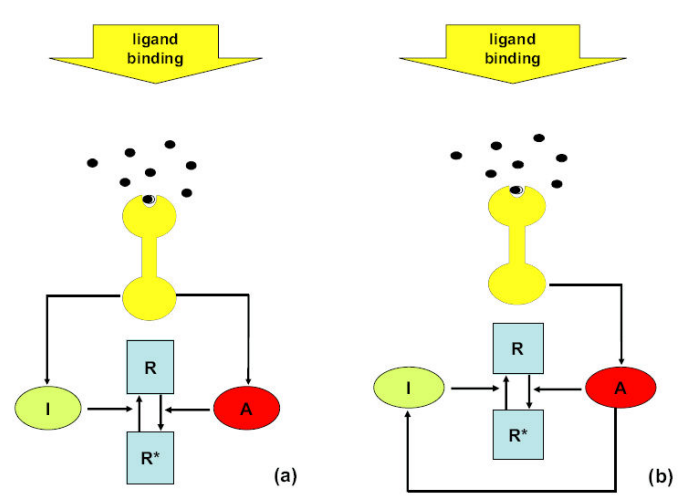

Fig. 1. Simplified Model of the chemotaxis system in the social amoebae Dictyostelium Discoideum.

circuit [20]. This, in turn, led researchers to focus on the task of identifying network topologies capable of implementing integral action and consistent with the known molecular proteins involved in chemotaxis. Below, we illustrate the use of our methodology in the discrimination between a number of equally plausible such topologies.

\section{B. A chemotaxis Model}

A number of mathematical models that describe the chemoattractant-induced response in Dictyostelium cells are available in the literature. Here, we describe a model that has been recently suggested to explain the basic architecture of the adaptation mechanism that is experimentally observed in Dictyostelium upon induction with a step function of chemoattractant [19]. The model consists of a response regulator (which eventually determines the rotation pattern of the flagellar motors) that can exist in one of two states: active $\left(R^{*}\right)$ and inactive $(R)$, such that its total number is constant $R_{T}=R(t)+R^{*}(t)$. This regulator is activated through the action of enzyme $A$ and inactivated through the action of enzyme $I$ (see Figure 1(a)). Using differential equations, one can describe the rate of change of $R^{*}$ as

$$
\begin{aligned}
\frac{d R^{*}(t)}{d t} & =-k_{-r} I(t) R^{*}(t)+k_{r} A(t) R(t) \\
& =-\left(k_{-r} I(t)+k_{r} A(t)\right) R^{*}(t)+k_{r} A(t) R_{T}
\end{aligned}
$$

where $k_{-r}$ and $k_{r}$ are forward and backward rate constants. Assume enzymes $A$ and $I$ are in turn regulated by an external signal $S$, proportional to chemoattractant concentration

$$
\begin{aligned}
\frac{d A(t)}{d t} & =-k_{-a} A(t)+k_{a} S(t) \\
\frac{d I(t)}{d t} & =-k_{-i} I(t)+k_{i_{1}} S(t)
\end{aligned}
$$

$k_{-a}, k_{a}, k_{-i}$ and $k_{i_{1}}$ are again rate constants. We will refer to this model as $\mathrm{A}$, and note that it possesses a unique equilibrium of active regulators at

$$
R^{*}(\infty)=\frac{k_{r} A(\infty) / I(\infty)}{k_{-r}+k_{r} A(\infty) / I(\infty)} R_{T}=\frac{k_{r} \frac{k_{A}}{k_{I}}}{k_{-r}+k_{r} \frac{k_{A}}{k_{I}}} R_{T}
$$


which is independent of chemoattractant concentration, hence identical before and after stimulus. This in turn implies that this model is perfectly rejecting step inputs, and thereby displaying the perfect adaptation characteristic of Dictyostelium chemotaxis.

\section{Other Perfectly Adapting Systems}

Although the model above is able to reproduce various properties of Dictyostelium chemotaxis such as perfect adaption, there is no direct experimental evidence that it is the actual topology implemented in the system. In fact, there is a number of other network topologies that use the same molecular players (i.e., $A, R, R^{*}$ and $I$ ) and also exhibit perfect adaptation. For example, if the inhibitory molecule $I$ is activated through the indirect action of the activator $A$ rather than through direct activation by ligand binding, the resulting system (Figure 1(b)), referred to as Model B, is described by the set of equations

$$
\begin{aligned}
\frac{d A(t)}{d t} & =-k_{-a} A(t)+k_{a} S(t) \\
\frac{d I(t)}{d t} & =-k_{-i} I(t)+k_{i_{2}} A(t) \\
\frac{d R^{*}(t)}{d t} & =-k_{-r} I(t) R^{*}(t)+k_{r} A(t) R(t) \\
& =-\left(k_{-r} I(t)+k_{r} A(t)\right) R^{*}(t)+k_{r} A(t) R_{T} .
\end{aligned}
$$

This model also perfect adapts to step inputs. Which model, A or B, is actually used by Dictyostelium to chemotax?

\section{Model Discrimination in Dictyostelium CHEMOTAXIS}

In this section we use the algorithms developed in Section III to design initial conditions that distinguish between models 1 and 2 developed in Section IV for Dictyostelium Discoideum Chemotaxis.

For notational convenience, we use $x=\left[R^{*}, A, I\right]$ to denote the state vector and $u=\left[S, R_{T}\right]$ to denote the input vector. By $x_{i j}$ we denote the $j$ th element of the state vector for model $i$, and similarly for $u_{i j}$. To determine the parameters for models $\mathrm{A}$ and $\mathrm{B}$, we assume that we have obtained time series data for the molecular species $R^{*}, A$, and $I$. It is common practice to determine kinetic parameters by fitting models to such data. Here, we use a profile of molecular species as given in Figure 2A. Fitting the models to such "data" generates the parameters: $k_{-a}=2$, $k_{-r}=1, k_{a}=3, k_{-i}=0.1, k_{i_{1}}=1$ and $k_{i_{2}}=$ $2 / 3$ for the models. Notice that we use "noiseless" data here, and therefore obtain perfect fits. However, in the most general case, the data would contain measurement noise and uncertainty, and therefore the fitting would ideally generate ranges of parameters rather than nominal values. In this case, robust model discrimination for these parameter ranges is needed. We will report on these cases elsewhere. Figure 2A can also be interpreted as the evolution of the two systems from a random initial condition. We are now well positioned to determine the initial conditions that would maximize the difference between the response of the two systems.
To answer this question, we apply our results to the problem of choosing initial conditions which, with no additional input perturbation from steady state (i.e. no addition of ligands), would maximize

$$
\int_{0}^{\infty} \sum_{i=1}^{3}\left(x_{1 i}-x_{2 i}\right)^{2} d t
$$

The basal input is set to $\left[S, R_{T}\right]=[0.2,0.7667]$. We first linearize our system around the equilibrium, and use the results of section II-C. The unit-norm direction that would maximize the above function is obtained as $x_{11}(0)=x_{21}(0)=0$, $x_{12}(0)=x_{22}(0)=1$, and $x_{13}(0)=x_{23}(0)=0$. Notice that these values correspond to the magnitude of the best perturbations of the states from the equilibrium achieved for the basal input $\left[S, R_{T}\right]=[0.2,0.7667]$. We similarly perform the same computations for the nonlinear systems using the results of section III. The unit-norm direction that would maximize the above function is obtained as $x_{11}(0)=x_{21}(0)=0.0137, x_{12}(0)=x_{22}(0)=0.9975$, and $x_{13}(0)=x_{23}(0)=0.0688$. These values are close to those obtained from the linearization of the system. Therefore, linearization provides important information in that respect, with a significantly reduced computational burden. The evolution of the states in Models A and B from the initial condition generated by the calculations related to the nonlinear system are plotted in Figure 2B.

A crucial observation out of these trajectories is that the initial conditions maximizing the difference between the two plausible models also generated a qualitative difference in the behavior of some of the state variables. For example, one notices that for Model B, the inhibitor $I$ ( $I_{2}$ in Figure $\left.2 \mathrm{~B}\right)$ has a biphasic response when the system is left from the computed "best" initial conditions. In this case, $I$ first decreases then increases back to finally converge to its equilibrium value. In contrast, $I$ generated by Model A does not show any such behavior for the initial conditions computed. This result appeals to our intuition about the system. In Model B, $I$ is chained to $A$. Therefore $I$ decreases as $A$ increases, and then strives for its own equilibrium once $A$ reaches its own steady-state. In model A, $I$ only responds to its own dynamics and is not chained to any other variables. This difference in the qualitative behavior was made the most salient through the choice of the appropriate initial conditions, as random initial condition as in Figure 2A would shadow this behavior. Indeed, one can postulate that if an experiment were performed, and $I$ measured as a function of time as it relaxes back to equilibrium from those "optimal" initial conditions, discrimination between the two models can be made possible based on the trends in the behavior of $I$.

\section{CONCLUSiOnS}

In this paper we have developed algorithmic tools for designing experiments that would distinguish between models of biochemical reaction networks. Specifically, we focused on the problem of constructing initial condition stimuli that generate maximum difference in the $L_{2}$ norm of the output of these networks. We further illustrated the use of this methodology in a plausible biological setting. 


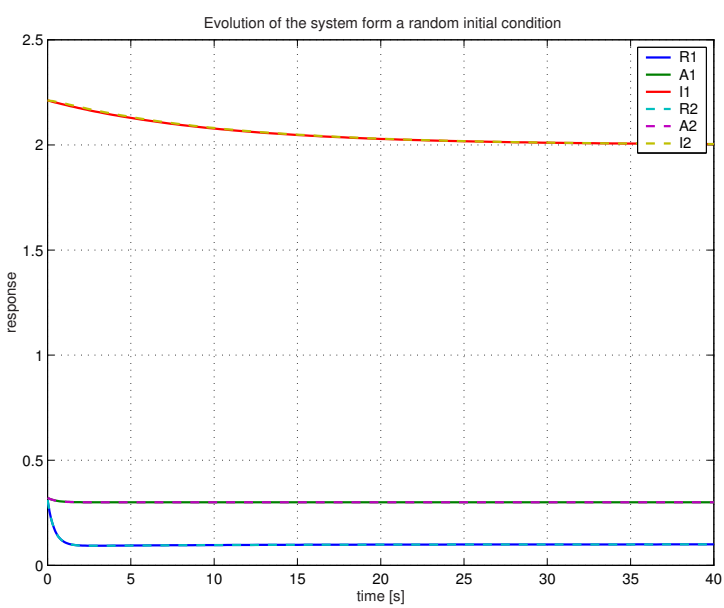

a

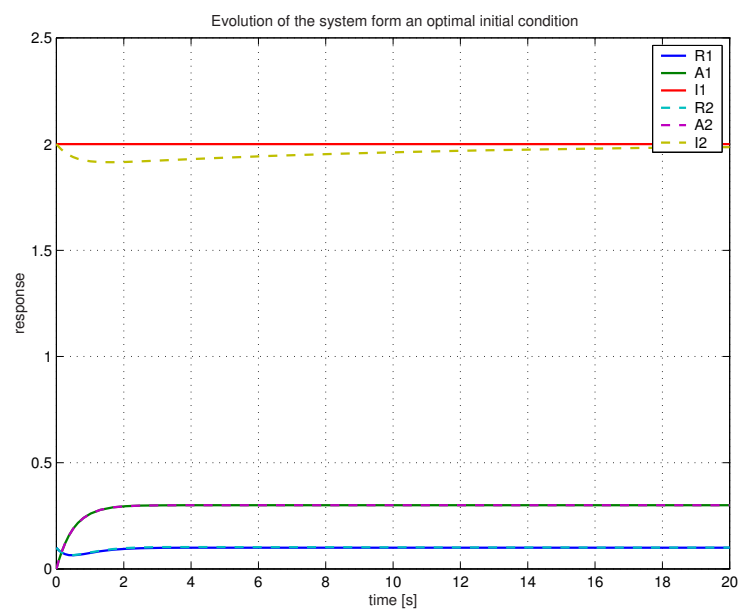

b

Fig. 2. Simulation of Models A and B when left from a random initial condition (a) and the 'best' unit-norm initial condition (b).

Although mathematically appealing, this work is only the first step in "closing the loop" between experiments and modeling. Many challenges remain: How can a mathematical quantity such as initial conditions be implemented in an experimental setting? Can we design 'optimal inputs' in the same way, so that they are experimentally achievable? In fact, it may be the case that some results of the algorithmic procedure do not correspond to specific experimental procedures that could be followed. Under these circumstances, further iterations may be necessary as such constraints must be explicitly taken into account. Rooting these algorithms in a sound biological reality will be a challenge, but it will undeniably be the only road to generating useful outcomes.

The data obtained from experiments that are "designed" in this way can be used to invalidate one, or even more models for the system. The invalidation would be done using tools that were developed in, say [9].

Lastly, we mention that systems with uncertainties can also be handled in the framework proposed in this paper. For example, some reactions in the proposed network models for the biological system would be allowed to have uncertain rate constants. In this case, the experiments have to be designed so as to discriminate between the different networks for all parameter values, in a 'robust' way.

\section{REFERENCES}

[1] B. H. Chen and S. P. Asprey, "On the design of optimally informative dynamic experiments for model discrimination in multiresponse nonlinear situations," Ind. Eng. Chem. Res., vol. 42, pp. 1379-1390, 2003.

[2] T. Chevalier, I. Schreiber, and J. Ross, "Toward a systematic determination of complex reaction mechanisms," J. Phys. Chem., vol. 97, no. 26, pp. 6776-6787, 1993.

[3] E. J. Crampin, S. Schnell, and P. E. McSharry, "Mathematical and computational techniques to deduce complex biochemical reaction mechanisms," Progress in Biophysics and Molecular Biology, vol. 86, pp. 77-112, 2004.

[4] K. G. Gadkar, R. Gunawan, and F. J. Doyle III, "Iterative approach to model identification of biological networks," BMC Bioinformatics, vol. 6 , p. $155,2005$.

[5] B. N. Kholodenko, A. Kiyatkin, F. J. Bruggeman, E. D. Sontag, H. V. Westerhoff, and J. B. Hoek, "Untangling the wires: A strategy to trace functional interactions in signalling and gene networks," Proceedings of the National Academy of Sciences, vol. 99, no. 20, pp. $12841-$ $12846,2002$.

[6] A. Kremling, S. Fischer, K. Gadkar, F. J. Doyle III, T. Sauter, E. Bullinger, F. Allgöwer, and E. D. Gilles, "A benchmark for methods in reverse engineering and model discrimination: Problem formulation and solutions," Genome Research, vol. 14, pp. 1773-1785, 2004.

[7] P. A. Parrilo, "Structured semidefinite programs and semialgebraic geometry methods in robustness and optimization," Ph.D. dissertation, Caltech, Pasadena, CA, 2000, available at http://www.control.ethz.ch/ parrilo/pubs/.

[8] M. Postma, J. Roelofs, J. Goedhart, T. W. G. Gadella, A. J. Visser, and P. j. Haatsert, "Uniform camp stimulation of dictyostelium cells induces localized patches of signal transduction and pseudopodia," Molecular Biology of the Cell, vol. 14, pp. 5019-5027, 2003.

[9] S. Prajna, "Barrier certificates for nonlinear model validation," Automatica, vol. 42, no. 1, pp. 117-126, 2006.

[10] S. Prajna, A. Papachristodoulou, and P. A. Parrilo, "SOSTOOLS Sum of Squares Optimization Toolbox, User's Guide," 2002, available at http://www.cds.caltech.edu/sostools.

[11] J. Ross, I. Schreiber, and M. O. Vlad, Determination of Complex Reaction Mechanisms: Analysis of Chemical, Biological and Genetic Networks. Oxford University Press, Inc., 2006.

[12] M. K. Stephen Yeung, J. Tegnér, and J. J. Collins, "Reverse engineering gene networks using singular value decomposition and robust regression," Proceedings of the National Academy of Sciences, vol. 99, no. 9, pp. 6163-6168, 2002.

[13] J. F. Sturm, "Using SeDuMi 1.02, a MATLAB toolbox for optimization over symmetric cones," Optimization Methods and Software, vol. 11-12, pp. 625-653, 1999, available at http: //fewcal.kub.nl/sturm/software/sedumi.html.

[14] M. Thelen, "Dancing to the tune of chemokines," Nature Immunology, vol. 2, pp. 129-134, 2001.

[15] W. Vance, A. Arkin, and J. Ross, "Determination of causal connectivities of species in random networks," Proceedings of the National Academy of Sciences, vol. 99, no. 9, pp. 5816-5821, 2002.

[16] G. H. Wadhams and J. P. Armitage, "Making sense of it all: Bacterial chemotaxis," Nature Reviews Molecular Cell Biology, vol. 5, pp. 1024-1037, 2004.

[17] J. C. Willems, "Dissipative dynamical systems. I. General theory," Arch. Rational Mech. Anal., vol. 45, pp. 321-351, 1972.

[18] — , "Dissipative dynamical systems. II. Linear systems with quadratic supply rates," Arch. Rational Mech. Anal., vol. 45, pp. 352393, 1972.

[19] L. Yang and P. A. Iglesias, "Positive feedback may cause the biphasic response observed in the chemoattractant-induced response of dictyostelium cells," Systems \& Control Letters, vol. 55, pp. 329-337, 2006.

[20] T. M. Yi, Y. Huang, M. I. Simon, and J. Doyle, "Robust perfect adaptation in bacterial chemotaxis through integral feedback control," Proceedings of the National Academy of Sciences, USA, vol. 97, pp. 4649-4653, 2000. 